\title{
Generalised Reliability On Hydro-Geo Objects
}

\author{
Wang Ya-jun ${ }^{*}, 1$ and Wang Jun ${ }^{2}$ \\ ${ }^{1}$ School of Maritime and Civil Engineering, Zhejiang Ocean University, Zhoushan, Zhejiang 316000, P. R. China \\ ${ }^{2}$ College of Architecture and Civil Engineering, Wenzhou University, Wenzhou, Zhejiang 325035, P. R. China
}

\begin{abstract}
This study established the fuzzy logic modeling of the stochastic finite element method based on the first-order approximation theorem. Fuzzy mathematical models of safety repertories were incorporated into the stochastic finite element method to analyze the stability of embankments and foundations in order to describe the fuzzy failure procedure for the random safety performance function. The fuzzy models were developed with membership functions with half depressed gamma distribution, half depressed normal distribution, and half depressed echelon distribution. The result shows that the middle region of the dike is the principal zone of concentrated failure due to local fractures. There is also some local shear failure on the dike crust. This study provides a referential method for solving complex multi-uncertainty problems in engineering safety analysis.
\end{abstract}

Keywords: Approximation algorithm, stochastic finite element method, fuzzy membership, dike reliability.

\section{INTRODUCTION}

Stability research on geo-structures, which has undergone a historical progression from the safety factor $K$ analysis phase to the reliability $J C$ application phase, has now entered a hybrid theoretical research period (Yang and Zhao 1999; Lü and Feng 1997) [1-2] in which probabilistic theorems and fuzzy mathematics are introduced into general numerical algorithms. Chinese scholars such as Chen and Liu (1990) [3] and Wang and Chen (1996) [4] offer some original achievements in this area. Though there is an essential difference between fuzziness and randomness of engineering phenomena, the fuzziness and randomness both belong to generalized uncertainty and infiltrate each other. According to the definition of the probability theory, random phenomena and their probability might be deterministic or fuzzy. Thus, fuzzy probability is formed. Based on these facts, geo-engineering subjects should be investigated comprehensively with fuzzy mathematical theory and the probabilistic analytical theorem.

Safety of structures is predominated by generalized loading and resistance. Therefore, generalized safety characteristics of structures can be simulated from dual perspectives. One is the fuzziness of structure strength and loading, which can be studied with the definition of relevant fuzzy membership function, and the other is fuzzy deduction of structure working behavior, which, instead of arbitrary subjectivities, is derived from the fact that work behavior of structures often shows intrinsic fuzzy characteristics in a state between safety and failure. The fuzzy characteristics can be simulated with fuzzy numerical techniques, i.e., softening techniques. With fuzzy softening techniques, the

*Address correspondence to this author at the School of Maritime and Civil Engineering, Zhejiang Ocean University, Zhoushan, 316000, P. R. China; Tel:15858052791; E-mail: aegis68004@163.com work behavior of structures is translated as a fuzzy event expressed by a generalized membership function $M$ that is a fuzzy subset submitting to state space of structures. In terms of deduction of structures' working behavior, there are two approaches for practical engineering: the fuzzy definition of the safety margin and the fuzzy definition of the safety ratio. In this study, the fuzzy processing of embankment working behavior is carried out with first-order stochastic approximation and the harmonious finite element (HFE) theorem, by which numerical simulation of fuzzy-stochastic uncertainties of the embankment system of the Yangtze River in the southern Jingzhou zone of China is realized.

The theoretical model described here has been applied to the calculation of fuzzy random generalized reliability of the main embankment of the Yangtze River in the southern Jingzhou zone of China. The main embankment of the Yangtze River in the southern Jingzhou zone plays a significant role in flood control of the Yangtze River Valley. Its distinguished characteristics, such as its great span length and complicated geological strata, certify the substantial value of this research.

\section{HARMONIOUS FINITE ELEMENT (HFE) FIRST- ORDER STOCHASTIC APPROXIMATION ALGORITHM}

As for the conventional finite element method, the governing equation, on the basis of the minimum potential energy principle, can be formulated as (Zhuo 2000) [5].

$$
\Pi_{\mathrm{p}}=\frac{1}{2} \delta^{\mathrm{T}} \boldsymbol{K} \delta-\delta^{\mathrm{T}} \boldsymbol{Q}, \quad \boldsymbol{K} \delta=\boldsymbol{Q}, \quad \boldsymbol{Q}=\int_{v} \boldsymbol{N}^{\mathrm{T}} \boldsymbol{f} \mathrm{d} v+\int_{s} \boldsymbol{N}^{\mathrm{T}} \boldsymbol{p} \mathrm{d} s
$$

where $\Pi_{\mathrm{p}}$ is the total potential energy, $\delta$ is the generalized displacement array, $\boldsymbol{K}$ is the stiffness matrix, $\boldsymbol{Q}$ is the 
equivalent nodal load array, $\boldsymbol{N}$ is the shape function interpolation matrix, $\boldsymbol{f}$ is the body force array, $\boldsymbol{p}$ is the surface load array, $v$ is the spatial domain, and $s$ is the surface domain. In practical engineering problems, $\boldsymbol{K}$ and $\boldsymbol{Q}$ are usually the functions of stochastic variable vector $\boldsymbol{X}=\left\{x_{1}, x_{2} \cdots x_{n}\right\}$. So, should be deduced as a function of $\boldsymbol{X}=\left\{x_{1}, x_{2} \cdots x_{n}\right\}: \boldsymbol{\delta}=\boldsymbol{\delta}\left(x_{1}, x_{2} \cdots x_{n}\right)$. There have been many numerical algorithms that treat the randomness of $\boldsymbol{X}$ (Lü and Feng 1997; Chen and Liu 1990; Wang and Chen 1996; Zhuo 2000; Christian and Baecher 1999) [2-6]. The first-order approximation theorem is applied here to expand the $\boldsymbol{\delta}$ into a Taylor series around the mean values of $\boldsymbol{X}$ (Yang and Zhao 1999; Lü and Feng 1997)[1, 2]. In this study, the linear first-order expansion was adopted. The expectation of the generalized displacement array $\boldsymbol{\delta}$ can be expressed in the following equation:

$$
\begin{aligned}
& E(\delta)=E\left[\delta\left(x_{1}, x_{2}, \cdots x_{n}\right)\right]+ \\
& \frac{\partial \delta}{\partial \boldsymbol{X}} \cdot\left\{\left(x_{1}-\overline{x_{1}}\right),\left(x_{2}-\overline{x_{2}}\right), \cdots\left(x_{n}-\overline{x_{n}}\right)\right\}^{\mathrm{T}} \\
& =\delta\left(\overline{x_{1}}, \overline{x_{2}}, \cdots, \overline{x_{n}}\right)
\end{aligned}
$$

where $E(\delta)$ is the mathematical expectation of $\delta$ and $\overline{x_{i}}(i=1,2, \cdots, n)$ is the even value of an algorithm model variable. When the nodal value of the generalized displacement array is expressed as $\delta_{i}(i=1,2, \cdots, m)$, with $m$ representing the node number of the corresponding numerical model, we have

$$
\begin{aligned}
& \mathrm{V}_{\mathrm{ar}}\left(\delta_{i}\right)=E\left\{\left[E\left(x_{i}\right)-\overline{x_{i}}\right]^{2}\right\} \\
& =E\left\{\left[\sum_{k=1}^{n}\left(x_{k}-\overline{x_{k}}\right)\left(\frac{\partial \delta_{i}}{\partial x_{k}}\right)\right]^{2}\right\} \\
& =\sum_{k=1}^{n} \sum_{l=1}^{n}\left(\frac{\partial \delta_{i}}{\partial x_{k}}\right)\left(\frac{\partial \delta_{i}}{\partial x_{l}}\right) E\left[\left(x_{k}-\overline{x_{k}}\right) \times\left(x_{l}-\overline{x_{l}}\right)\right] \\
& =\sum_{k=1}^{n} \sum_{l=1}^{n}\left(\frac{\partial \delta_{i}}{\partial x_{k}}\right)\left(\frac{\partial \delta_{i}}{\partial x_{l}}\right) \mathrm{C}_{\text {ov }}\left(x_{k}, x_{l}\right)
\end{aligned}
$$

where $\mathrm{V}_{\mathrm{ar}}\left(\delta_{i}\right)$ is the variance of $\delta_{i}, \mathrm{C}_{\mathrm{ov}}\left(x_{k}, x_{l}\right)$ is the covariance of $x_{k}$ and $x_{l}$, and $\frac{\partial \delta_{i}}{\partial x_{k}}$ and $\frac{\partial \delta_{i}}{\partial x_{l}}$ are the partial derivatives of generalized nodal displacement $\delta_{i}$ with respect to $x_{k}$ and $x_{l}$ at the mean value of $\boldsymbol{X}$. Similarly, in the harmonious finite element algorithm, the stress vector $\sigma$ is also a function of random variable $\boldsymbol{X}$ : $\boldsymbol{\sigma}=\boldsymbol{\sigma}\left(x_{1}, x_{2} \cdots x_{n}\right)$. When the element stress is expressed as $\sigma_{i}=\sigma_{i}\left(x_{1}, x_{2} \cdots x_{n}\right)$ , its corresponding variance as well as covariance can be formulated as follows (An et al. 2002) [7]:

$$
\begin{aligned}
& \mathrm{V}_{\mathrm{ar}}\left(\sigma_{i}\right)=\sum_{k=1}^{n} \sum_{l=1}^{n}\left(\frac{\partial \sigma_{i}}{\partial x_{k}}\right)\left(\frac{\partial \sigma_{i}}{\partial x_{l}}\right) \mathrm{C}_{\mathrm{ov}}\left(x_{k}, x_{l}\right) \mathrm{C}_{\mathrm{ov}}\left(\sigma_{i}, \sigma_{j}\right) \\
& =\sum_{k=1}^{n} \sum_{l=1}^{n}\left(\frac{\partial \sigma_{i}}{\partial x_{k}}\right)\left(\frac{\partial \sigma_{j}}{\partial x_{l}}\right) \mathrm{C}_{\mathrm{ov}}\left(x_{k}, x_{l}\right)
\end{aligned}
$$

For further information on Eq. 4, one can refer to related work (Christian and Bacher 1999; An et al., 2002; Xu 2001) [6-8] that has offered the key algorithms for $\partial \sigma_{1} / \partial x_{k}$, $\partial \sigma_{2} / \partial x_{k}$, and $\partial \tau_{\max } / \partial x_{k}$. Based on the content of the cited research, variance and covariance values of normal stress and maximal shear stress can be obtained from Eq. 4 . Moreover, because the influence of structure body force is of great importance to stochastic numerical models for geoengineering (Xu 2001) [8], the random vector $\boldsymbol{Q}$ in Eq. 1 is also a function of $\boldsymbol{X}$.

\section{RANDOM MODEL FOR LOCAL FAILURE OF HETEROGENEOUS STRUCTURE}

Analyzed here is the local failure probability of an isotropic heterogeneous geo-structure without a predominant discontinuous slip crack interlayer. The failure probability under shear deformation and tensile deformation was calculated with the Mohr-Coulomb failure criterion. The strength characteristics of geo-material mainly include cohesive force $c$ and internal friction angle $\phi$. Owing to the intrinsic stochastic discreteness of survey data of $c$ and $\phi, c$ and $\phi$ can be regarded as random variables (Chowdhury and Xu 1995; Guo and Wen 1992) [9,10]. Two principal safety reserve models are adopted in this study. They are the shear model $q_{\mathrm{s}}$ and the tensile model $q_{\mathrm{t}}$, whose expressions are as follows:

$$
\begin{aligned}
& q_{\mathrm{s}}=\tau_{\mathrm{f}}-\tau_{\max } \\
& =c \cos \phi+0.5\left(\sigma_{1}+\sigma_{2}\right) \sin \phi-0.5\left(\sigma_{1}-\sigma_{2}\right) \\
& q_{\mathrm{t}}=\sigma_{2}
\end{aligned}
$$

where $\tau_{\mathrm{f}}$ and $\tau_{\max }$ are, respectively, the distance from the center of the Mohr stress circle to the limiting failure line and the radius of the stress circle, and $\sigma_{1}$ and $\sigma_{2}$ are the maximum principal stress and intermediate principal stress, respectively.

It can be inferred from the foregoing analysis that $q_{\mathrm{s}}$ and $q_{\mathrm{t}}$ are stochastic variables. Therefore, their expectations and variances are formulated by the following equations:

$$
\begin{aligned}
& \mathrm{E}\left(q_{\mathrm{s}}\right)=\mathrm{E}(c) \cos [\mathrm{E}(\phi)]+0.5\left[\mathrm{E}\left(\sigma_{1}\right)+\mathrm{E}\left(\sigma_{2}\right)\right] \cdot \\
& \sin [\mathrm{E}(\phi)]-0.5\left[\mathrm{E}\left(\sigma_{1}\right)-\mathrm{E}\left(\sigma_{2}\right)\right] \\
& \mathrm{E}\left(q_{\mathrm{t}}\right)=\mathrm{E}\left(\sigma_{2}\right) \\
& \mathrm{V}_{\mathrm{ar}}\left(q_{\mathrm{s}}\right)=\left(\frac{\partial q_{\mathrm{s}}}{\partial c}\right)^{2} \mathrm{~V}_{\mathrm{ar}}(c)+\left(\frac{\partial q_{\mathrm{s}}}{\partial \phi}\right)^{2} \mathrm{~V}_{\mathrm{ar}}(\phi)+ \\
& \left(\frac{\partial q_{\mathrm{s}}}{\partial c}\right)\left(\frac{\partial q_{\mathrm{s}}}{\partial \phi}\right) \mathrm{C}_{\mathrm{ov}}(c, \phi)+\sum_{k=1}^{n}\left(\frac{\partial q_{\mathrm{s}}}{\partial x_{k}}\right)\left(\frac{\partial q_{\mathrm{s}}}{\partial c}\right) \mathrm{C}_{\mathrm{ov}}\left[x_{k}, c\right]+
\end{aligned}
$$


$\sum_{k=1}^{n}\left(\frac{\partial q_{\mathrm{s}}}{\partial x_{k}}\right)\left(\frac{\partial q_{\mathrm{s}}}{\partial \phi}\right) \mathrm{C}_{\mathrm{ov}}\left[x_{k}, \phi\right]+$

$\sum_{k=1}^{n} \sum_{l=1}^{n}\left(\frac{\partial q_{\mathrm{s}}}{\partial x_{k}}\right)\left(\frac{\partial q_{\mathrm{s}}}{\partial x_{l}}\right) \mathrm{C}_{\mathrm{ov}}\left[x_{k}, x_{l}\right]$

$\mathrm{V}_{\mathrm{ar}}\left(q_{\mathrm{t}}\right)=\sum_{k=1}^{n} \sum_{l=1}^{n}\left(\frac{\partial \sigma_{2}}{\partial x_{k}}\right)\left(\frac{\partial \sigma_{2}}{\partial x_{l}}\right) \mathrm{C}_{\mathrm{ov}}\left(x_{k}, x_{l}\right)$

\section{FUZZY STOCHASTIC FAILURE MODEL OF HETEROGENEOUS STRUCTURE}

With the Mohr-Coulomb failure criterion and limiting equilibrium theorem, the safety factor of a heterogeneous geo-structure can be defined as follows:

$F_{\mathrm{s}}=\frac{E\left(\tau_{\mathrm{f}}\right)}{E\left(\tau_{\max }\right)}$

$=\frac{E(c) \cos [E(\phi)]+0.5\left[E\left(\sigma_{1}\right)+E\left(\sigma_{2}\right)\right] \sin [E(\phi)]}{0.5\left[E\left(\sigma_{1}\right)-E\left(\sigma_{2}\right)\right]}$

The reliability index is used to evaluate the random working behavior of geo-structures (Hassan and Wolff 1999; Low et al. 1998) [11,12]. Based on the assumption that $q_{\mathrm{s}}$ and $q_{\mathrm{t}}$ submit to Gaussian distribution, the reliability index can be expressed as $\beta=\mu_{q_{\mathrm{s}}} / \sigma_{q_{\mathrm{s}}}$, where $\mu_{q_{\mathrm{s}}}$ and $\sigma_{q_{\mathrm{s}}}$ are, respectively, the even value and mean-square deviation of $q_{\mathrm{s}}$.

The general failure probability $P_{\mathrm{f}}$ of geo-structures can be formulated as follows:

$$
P_{\mathrm{f}}=P(Z<0)=\int_{-\infty}^{0} \frac{1}{\sqrt{2 \pi} \sigma_{z}} \exp \left[-\frac{1}{2}\left(\frac{z-\mu_{z}}{\sigma_{z}}\right)^{2}\right] d z
$$

where $Z$ is the status function of geo-structures; $\sigma_{z}$ and $\mu_{z}$ are, respectively, the mean-square deviation and even value of the status function $Z$; and $z$ is the probabilistic integration variable whose span is determined by $Z$. The generalized membership function $M$ of geo-structure working behavior is expressed in Zadeh notation as Eq. 10, which, as softening technology, can realize the fuzzy formulation of the safety reserve model:

where $g(\boldsymbol{X})$ is the status function, $\mu_{M}[g(\boldsymbol{X})]$ is the membership, and $\Omega$ is the fuzzy coverage.

$M=\int_{\Omega} \frac{\mu_{M}[g(\boldsymbol{X})]}{\boldsymbol{X}}$

Based on the extension principle, the descriptions of failure probability and reliability index, $P_{\mathrm{f}}{ }^{*}$, the fuzzystochastic failure probability of geo-structure local deformation can be expressed as

$P_{\mathrm{f}}^{*}=\int_{-\infty}^{\infty} \frac{1}{\sqrt{2 \pi} \sigma_{q_{\mathrm{s}}}} \mu_{M}\left(q_{\mathrm{s}}\right) \exp \left[-\frac{1}{2}\left(\frac{q_{\mathrm{s}}-\mu_{q_{\mathrm{s}}}}{\sigma_{q_{\mathrm{s}}}}\right)^{2}\right] d q_{\mathrm{s}}$

where all parameters are defined based on the $q_{s}$ status function of geo-structures: $\sigma_{q_{\mathrm{s}}}$ and $\mu_{q_{\mathrm{s}}}$ are, respectively, the mean-square deviation and even value of the status function $q_{\mathrm{s}}$, and $\mu_{M}\left(q_{\mathrm{s}}\right)$ is the fuzzy-stochastic membership.

Furthermore, in order to better analyze the embankment slope failure model, the comprehensive safety ratio $R_{\mathrm{s}}$ is defined as (Wang 2004) [13].

$R_{\mathrm{s}}=\frac{q_{\mathrm{s}}}{0.5\left(\sigma_{1}-\sigma_{2}\right)}=\frac{\tau_{\mathrm{f}}-\tau_{\max }}{\tau_{\max }}=\frac{\tau_{\mathrm{f}}}{\tau_{\max }}-1$.

Uniting foregoing contents, the membership functions of three fuzzy models are shown in Table $\mathbf{1}$

\section{FUZZY STOCHASTIC NUMERICAL ALGORITHM APPLICATION TO EMBANKMENT STRUCTURE}

In this study, in order to investigate the random working behavior of the geotechnical structure, a typical section $580+500$ of the main embankment of the Yangtze River in the southern Jingzhou zone of China was analyzed with the local failure model using the fuzzy stochastic numerical algorithm.

Fig. (1) shows the cross section of the dike structure. The dike structure is divided into three stuff-fill zones whose

Table 1. Membership functions of three fuzzy models.

\begin{tabular}{|c|c|c|}
\hline Fuzzy models & Membership functions & \\
\hline Half depressed gamma distribution & $\mu_{\underline{M}}\left(R_{\mathrm{s}}\right)=\left\{\begin{array}{l}1 \\
\exp \left[-8.66 \times\left(R_{\mathrm{s}}+0.08\right)\right]\end{array}\right.$ & $\begin{array}{l}R_{\mathrm{s}} \leq-0.08 \\
R_{\mathrm{s}} \geq-0.08\end{array}$ \\
\hline Half depressed normal distribution & $\mu_{\underline{M}}\left(R_{\mathrm{s}}\right)=\left\{\begin{array}{l}1 \\
\exp \left[-108.3 \times\left(R_{\mathrm{s}}+0.08\right)^{2}\right.\end{array}\right.$ & $\begin{aligned} R_{\mathrm{s}} \leq- & 0.08 \\
& R_{\mathrm{s}} \geq-0.08\end{aligned}$ \\
\hline Half depressed echelon distribution & $\mu_{\underline{M}}\left(R_{\mathrm{s}}\right)=\left\{\begin{array}{l}1 \\
\frac{0.5-R_{\mathrm{s}}}{0.5+0.08}\end{array}\right.$ & $\begin{array}{r}R_{\mathrm{s}} \leq-0.08 \\
-0.08<R_{\mathrm{s}} \leq 0.5\end{array}$ \\
\hline
\end{tabular}


Table 2. Material properties of dike body.

\begin{tabular}{|c|c|c|c|c|c|}
\hline Zone & $\boldsymbol{E}(\mathbf{M P a})$ & $\boldsymbol{v}$ & $\boldsymbol{\rho ( \mathbf { 1 0 } ^ { \mathbf { 6 } } \mathbf { g } \cdot \mathbf { m } ^ { - \mathbf { 3 } } )}$ & $\boldsymbol{c}(\mathbf{k P a})$ & $\boldsymbol{\omega}\left(\mathbf{0}^{\mathbf{}}\right)$ \\
\hline \hline 1 & 4.1 & 0.3 & 1.41 & 15 & 18 \\
\hline 2 & 2.8 & 0.25 & 1.55 & 21.6 & 21 \\
\hline 3 & 14 & 0.1 & 1.64 & 0 & 31 \\
\hline
\end{tabular}

material parameters are shown in Table 2.

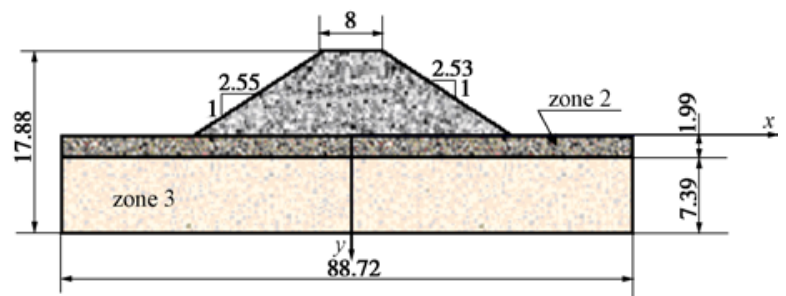

Fig. (1). Cross section of the dike structure (m).

Under gravitational body loading, the numerical model grid, whose boundary limit runs $19.4 \mathrm{~m}$ away from the upstream dike-ankle and $18.3 \mathrm{~m}$ away from the downstream dike-toe, has 275 elements and 174 nodes. It is assumed that the material parameters are statistically independent. Covariance matrixes of random parameters of three material zones are reduced to diagonal matrixes (Schweiger et al. 2001) [14] and the principal diagonal elements are the following:

$\operatorname{diog}\left(\mathrm{C}_{\mathrm{ov}_{1}}\right)=(0.50,0.24,0.30,0.44,0.17)$

$\operatorname{diog}\left(\mathrm{C}_{\mathrm{ov}_{2}}\right)=(0.32,0.24,0.30,0.14,0.17)$

$\operatorname{diog}\left(\mathrm{C}_{\mathrm{ov}_{3}}\right)=(0.15,0.24,0.13,0.44,0.21)$

Fig. (2) presents the deformed grid of a typical section under loading and it is evident that the stress concentration zone is in the middle region of embankment body. The latter displacement contour figures and stress contour figures (Fig. (3), Fig. (5), Fig. (7), and Fig. (8) demonstrate this fact as well.

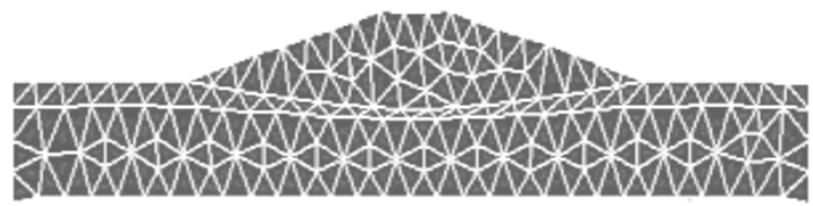

Fig. (2). Sketch of mean deformations of dam mesh.

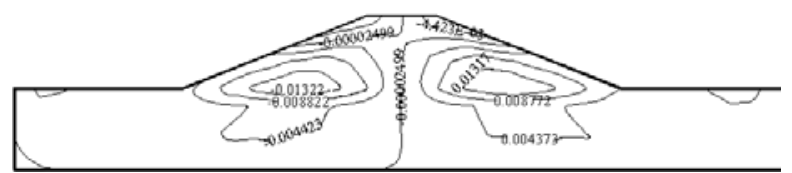

Fig. (3). Contours of mean of $x$-displacement in embankment section (m).

From Fig. (3) it can be deduced that the maximum gradient variation of the $x$ displacement accumulates in the middle region of the embankment system, which is consistent with monitoring information (Wang 2004) [13]. Furthermore, some cracks almost break through the whole dike crown on the local embankment sections. There are two reasons for this. The first is continual hydraulic scouring and random oscillation of the predominant waterline that can be summarized as external hydraulic influence, and the second is intrinsic stochastic characteristics. Sandy soil layers occupy most of the geo-space of the main embankment of the Yangtze River in the southern Jingzhou zone. The embankment foundation has a small expectation value of $y$ displacement and a high value of $E$. However, shear deformation occurs and a local seepage channel is formed in the middle region of the embankment system. They have often put the embankment system in danger. Therefore, the high probability of absolute collapse under embankmentbase erosion deformation must receive more attention. The displacement variance contours Fig. (4) and Fig. (6) show a small magnitude of variation of the stochastic displacement field, by which it can be deduced that the variation of the random displacement field has a low sensitivity to stochastic turbulence of material parameters.

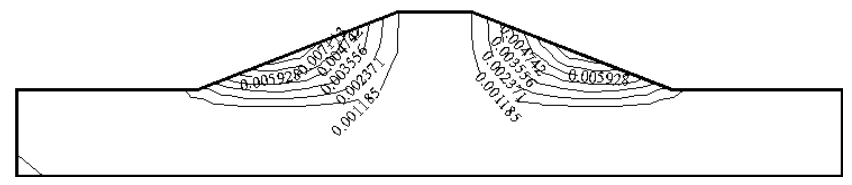

Fig. (4). Contours of variance of $x$-displacement in embankment section $\left(\mathrm{m}^{2}\right)$.

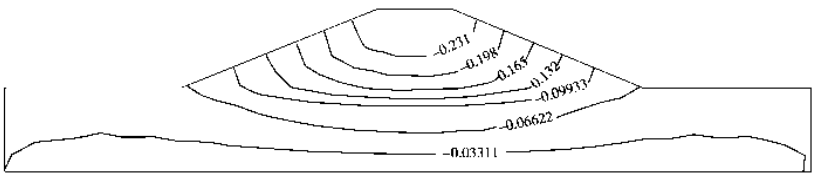

Fig. (5). Contour of mean of $y$-displacement in embankment section (m).

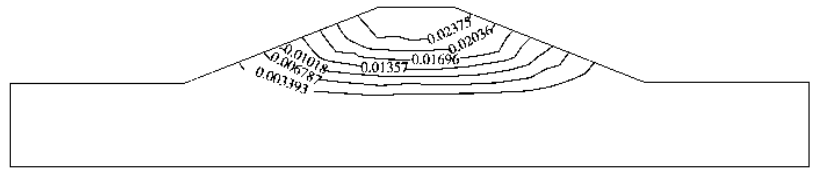

Fig. (6). Contour of variance of $y$-displacement in embankment section $\left(\mathrm{m}^{2}\right)$.

Random fluctuation of the stress field of the main embankment is shown in Figs. (7-10). The stress distribution shows great discreteness, and the stress variance is $10^{2}$ times larger than the displacement variance in some regions. The high sensitivity of the stress field to random material parameters aggravates the stress concentration. Related research (Christian and Baecher 1999) [6] shows that these characteristics are consistent with the stochastic calculation results of $q_{\mathrm{s}}$ and $q_{\mathrm{t}}$, and reveal potential danger in the local region of the embankment. Expectation values of the two principal safety reserve models are low enough to undermine 
partial geo-zones, and this is demonstrated by on-the-spot investigation of the main embankment of the Yangtze River in the southern Jingzhou zone.

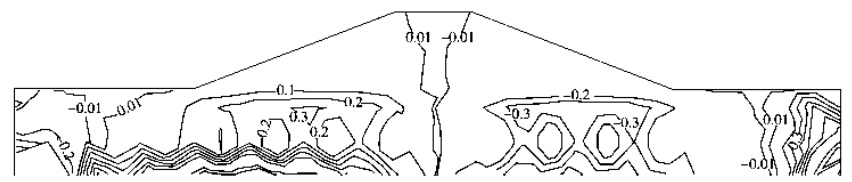

Fig. (7). Contours of mean of principal stress $\sigma_{1}$ on embankment section (MPa)

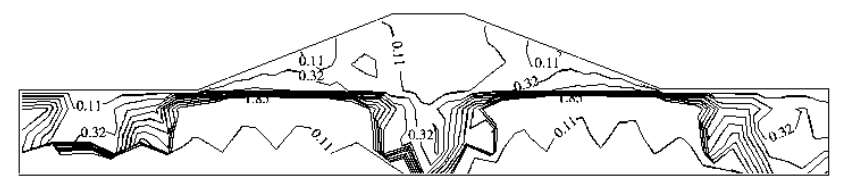

Fig. (8). Contours of variance of principal stress $\sigma_{1}$ on embankment section $\left(\mathrm{MPa}^{2}\right)$.

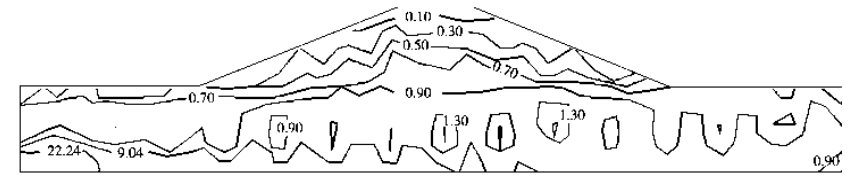

Fig. (9). Contours of mean of shear stress $\tau_{x y}$ on embankment section (MPa).

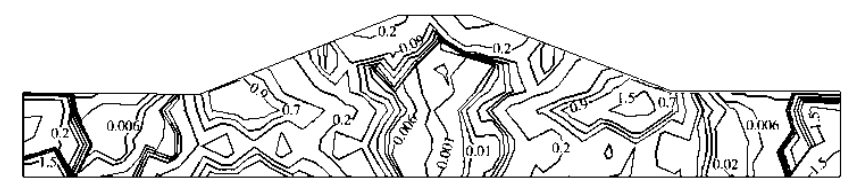

Fig. (10). Contours of variance of shear stress $\tau_{x y}$ on embankment section $\left(\mathrm{MPa}^{2}\right)$.

Like the displacement field and stress contour distribution, the safety factor $F_{\mathrm{s}}$ contour distribution (Fig. 11) shows the risk tendency of the dike middle region where $F_{\mathrm{s}}$ reaches its minimum. Meanwhile, the safety factor $F_{\mathrm{s}}$ also decreases in the dike crown because the magnitude of the shear model $q_{\mathrm{s}}$ is $10^{-2}$ in the dike crown. The small magnitude of the reliability index ( $\beta$ (Fig. 12) ) occupies most of the space of the dike middle region. Both Fig. (11) and Fig. (12) indicate that local failure mainly occurs in the dike middle region.

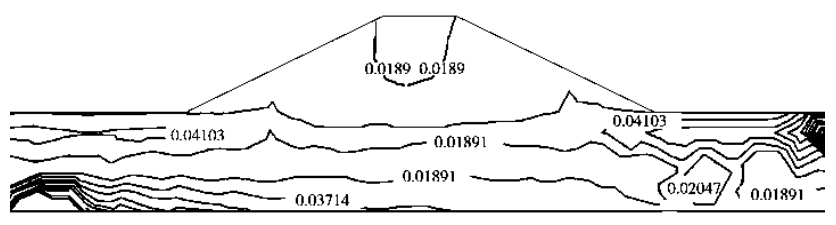

Fig. (11). Contours of safety factors $F_{\mathrm{s}}$ on embankment section.

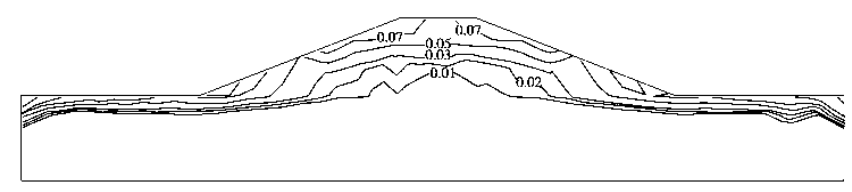

Fig. (12). Contours of reliability index $\beta$ on embankment section.

The main embankment of the Yangtze River in the southern Jingzhou zone is characterized by a strata structure and the dike middle region is in high danger due to stress concentration. The embankment crown absorbs more stress momentum than downstream and upstream. A sandy sediment substratum with a highly variable stress field becomes a dead zone where the embankment foundation is easily undermined when the exterior water level fluctuates greatly. Moreover, as Fig. (11) shows, the deterministic numerical analysis reflects mainly the outer layer working behavior whose reserve safety factor, calculated by $q_{s}$, only reaches a minor magnitude of $10^{-2}$. On the contrary, the

working behavior of the embankment system core layer is described by the failure probability contour distribution (Figs 13-15) and analyzed by three fuzzy stochastic mathematical models, the half depressed gamma distribution with failure probability $P_{\mathrm{f}}^{*}$, the half depressed normal distribution with failure probability $P_{\mathrm{fN}}^{*}$, and the half depressed echelon distribution with failure probability $P_{\mathrm{fE}}^{*}$. The conformity of random distribution characteristics of stress contours and the reliability index contours also indicate the dangerous working behavior of the dike body core layer. of embankment section for half depressed normal distribution.

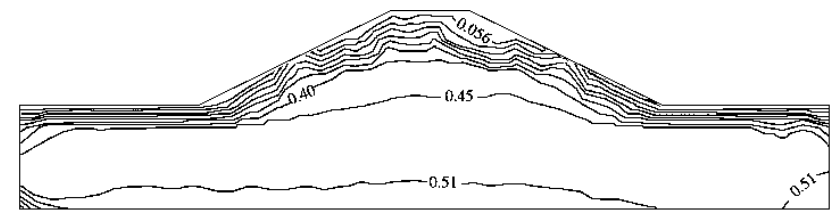

Fig. (13). Contours of local failure probability $P_{\mathrm{fN}}^{*}$.

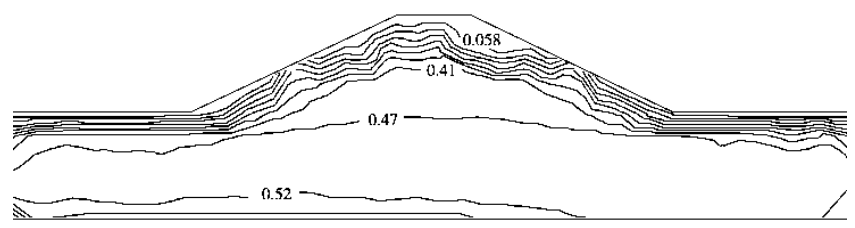

Fig. (14). Contours of local failure probability $P_{\mathrm{fE}}^{*}$ of embankment section for half depressed echelon distribution.

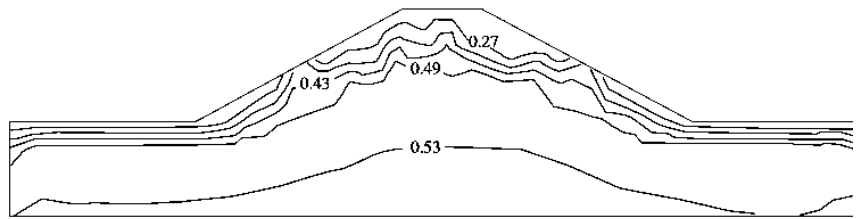

Fig. (15). Contours of local failure probability $P_{\mathrm{f}}^{*}$ of embankment section for half depressed gamma distribution.

\section{CONCLUSION}

(1) In contrast to the conventional finite element numerical algorithm, which can only calculate the deterministic vector fields including displacement and stress, the stochastic numerical algorithm is able to generate random vector fields: expectation value, variance and variation of displacement and stress.

(2) The first-order approximation algorithm offers a definite numerical model, and has superior calculation accuracy and effective applicability for random simulation with parameter variation less than 0.3 , though its numerical program development is complex. 
(3) Softening technology is able to realize the fuzzy formulation of the safety reserve model that can analyze the uncertainty of the geo-structure working behavior. Three fuzzy mathematical models have been introduced in this study to comprehensively study generalized vector field characteristics.

(4) The model calculation results indicate that the dike middle region is the principal concentrated failure zone, which is demonstrated by the on-the-spot investigation. Furthermore, there is also some local shear failure on the dike crust.

\section{CONFLICT OF INTEREST}

The authors confirm that this article content has no conflict of interest.

\section{ACKNOWLEDGEMENTS}

This work was supported by the National Natural Science Foundation of China (Grant No: 51109118), Zhejiang Provincial Natural Science Foundation of China (Grant No: LY14E090001), United Development Project Foundation from Zhejiang Ocean University and Wenzhou University (Grant No: 21188004113), United Development Project Foundation from Zhejiang Ocean University and Hydrochina Huadong Engineering Corporation (Grant No: 21188004013) and Young Teachers Improvement Project Fund of Zhejiang Ocean University (Grant No: 11042101512).

\section{REFERENCES}

[1] W. J. Yang and C. Z. Zhao, Reliability Theory of Civil Engineering Structures and Design, China Communication Press, Beijing: 1999, pp. 19-66. (in Chinese)

[2] Z. Z. Lü and Y. S. Feng, "The general reliability calculation method based on randomness and fuzziness for structure”, Acta
Mechanica Solida Sinica, vol. 18, no. 1, pp. 80-85, 1997. (in Chinese)

[3] Q. Chen and X. B. Liu, Stochastic Finite Element Method and its Engineering Applications, Southwest Jiaotong University Press, Chengdu: 1993. pp. 10- 93, (in Chinese)

[4] G. Y. Wang and S. X. Chen, Theory of Soft Design for Engineering Structural Systems and its Application, National Defense Industry Press, Beijing: 1996. pp. 23-79, (in Chinese)

[5] J. S. Zhuo, "Generalized Variational Principle in Elasto-Plastic Mechanics,” Hohai University Press, Nanjing: 2000. pp.23-96, (in Chinese)

[6] J. T. Christian and G. B. Baecher, "Point-estimate method as numerical quadrature”, Journal of Geotechnical and Geoenvironmental Engineering, vol. 125, no. 9, pp. 779-786, 1999.

[7] W. G. An, W. B. Zhu and X. C. Yan, "Application of stochastic finite element method to analysis of uncertainties", Journal of Harbin Technology University, vol. 23, no. 1, pp. 132-135, 2002. (in Chinese)

[8] Z. L. Xu, "Finite Element Method in Elastic Mechanics Problems," Hohai University Press, Nanjing: 2001. pp. 40-105, (in Chinese)

[9] R. N. Chowdhury, and D. W. Xu, "Geotechnical system reliability of slopes”, Reliability Engineering and System Safety, vol. 47, no. 3, pp. 141-151, 1995.

[10] X. M. Guo and X. L. Wen, "The stochastic finite element method for the mechanical analysis of rock masses", Journal of North China Institute of Water Conservancy and Hydroelectric Power, no. 1, pp. 44-51, 1992. (in Chinese)

[11] A. M. Hassan and T. F. Wolff, "Search algorithm for minimum reliability index of earth slopes", Journal of Geotechnical and Geoenvironmental Engineering, vol. 125, no. 4, pp. 301-308, 1999.

[12] B. K. Low, R. B. Gilbert and S. G. Wright, "Slope reliability analysis using generalized method of slices", Journal of Geotechnical and Geoenvironmental Engineering, vol. 124, no. 4, pp. 350-362, 1998.

[13] Y. J. Wang, "Application of generalized reliability method based on fuzzy-stochastic theorem in bank slope stability analysis", Geotechnical Engineering Technique, vol. 18, no. 5, pp. 217-223, 2004. (in Chinese)

[14] H. F. Schweiger, R. Thurner and R. Pöttler, "Reliability analysis in geotechnics with deterministic finite elements-Theoretical concepts and practical application”, The International Journal of Geomechanics, vol. 1, no. 4, pp. 389-413, 2001. 